\title{
Effect of mode of delivery on couples' sexual relationship during the postpartum period
}

\section{Original Article}

\author{
Sherine H. Abdel Rahman', Ayman A. Zeid', MonaEl Awady ${ }^{3}$ \\ ${ }^{1}$ Departments of Dermatology, Andrology and Venereology, ${ }^{3}$ Community Medicine, Benha \\ University, Benha, ${ }^{2}$ Department of Gynecology and Obstetric, El Mataryia Teaching \\ Hospital, Cairo, Egypt.
}

\begin{abstract}
Background: Although, sexual function is important in married couples' relationship, little is known about the intersexual relationship especially after delivery.

Objective: This is a cross-sectional study which aimed to assess and compare the impact of mode of delivery on sexual function of married couples and explore their sexual relationship after delivery.

Patients and Methods: This is a cross-sectional study which enrolled 400 couples during the postpartum period and were equally divided according to the mode of delivery into two groups: group I (200) included women who had a cesarean section and group II (200) had women who had vaginal delivery (VD) and their husbands. Each group was equally subdivided according to the number of deliveries (multiparous $=100$ and nulliparous $=100$ ). Sexual functions of men and women were assessed according to the female sexual function index and international index of erectile function 15 questionnaires, respectively.

Results: Couples in the cesarean section group showed better sexual functions than in the VD group with a correlation between international index of erectile function and female sexual function index domains.

Conclusion: VD, episiotomy, and multipara negatively affect female and male sexual domains.
\end{abstract}

Key Words: Cesarean section, episiotomy, female sexual function index, international index of erectile function, vaginal delivery.

Received: 11 March 2020, Accepted: 10 April 2020.

Corresponding Author: Sherine H. Abdel Rahman Ph.D, Departments of Dermatology, Andrology and Venereology, Benha University, Benha, Egypt, Tel.: 01223196080, E-mail: sherine1771@gmail.com.

ISSN: 2090-6048, 2019 Vol. 9, No.

\section{INTRODUCTION}

Couples' relationship is important in the transition phase to parenthood and in raising a child, especially during early childhood for the upbringing of a psychologically stable child. Couples' sexual relationship has a substantial impact on couples' relationship ${ }^{[1]}$ and childbirth is among the most important factors that influencessexualfunction ${ }^{[2]}$. Available data suggest that postpartum female sexual dysfunction (FSD) is common in many countries, although with inconsistent findings.

As many women are ignorant of the changes in theirsexualfunctions after childbirth and wonder if the mode of delivery has an impact on sexual dysfunction (SD), in addition to the inadequate knowledge about the sexual interrelationship between married couples especially after childbirth. Therefore, the current study was conducted to assess and compare the impact of mode of delivery on sexual function of married couples and explore their sexual relationship after delivery.

\section{PATIENTS AND METHODS}

This is a cross-sectional study which was conducted on 400 couples in the period from 6 months to 1-year postpartum after the last delivery. They were recruited from outpatient clinics of Obstetrics and Gynaecology Department and from the primary health-care centers and units from September 2018 to December 2019. After taking the approval of the ethics committee, the aim of the study was explained to all participants and an informed consent was obtained.

The 400 couples were equally divided according to the mode of delivery into two groups: group I (200) included women who had a cesarean section (CS) and group II (200) had women who had vaginal delivery (VD) and their husbands. Each group was equally subdivided according to the number of deliveries (multiparous $=100$ and nulliparous $=100$ ).

Only, premenopausal sexually active women and their spouses were enrolled into this study if they are not illiterate 
or not having any of the following items which might affect sexual function in both sexes: having a chronic disease (hypertension, diabetes, cardiac diseases, renal diseases, or hepatic diseases), or autoimmunediseaseorneoplasia. Women who were pregnant, lactating, taking any hormonal medication even as a contraceptive method, having postpartum depression, or with husbandshaving any type of SD were excluded from the study.

All participants were interviewed for sociodemographic data, duration of marriage, number of children, and for women, mode of delivery and if episiotomy was done to VD group II.

To assess female and male sexual functions, participants and their partners were asked to fill out an Arabic validated version questionnaire ${ }^{[3]}$ of female sexual function index (FSFI ${ }^{[4]}$ and international index of erectile function (IIEF-15) ${ }^{[5]}$, respectively. They were then asked to put the questionnaire in an envelope and seal it and leave it anonymously.

The FSFI is avalidated 19-item, self-administered, screening questionnaire that measures the aspects of female sexual function in six domains (desire, arousal, lubrication, orgasm, satisfaction, and pain).

The IIEF-15 is a validated brief self-administered 15 -item questionnaire that evaluates five domains in male sexual function (erectile and orgasmic function, sexual desire, intercourse, and overall satisfaction).

\section{Data analysis}

The sample size was calculated according to the equation:

$$
N=\frac{Z^{2} \cdot P Q}{E^{2}} .
$$

Where $\mathrm{N}=$ minimal sample size, $\mathrm{Z}=1.96, \mathrm{P}=40-45 \%$, and $\mathrm{E}=0.05$

If $\mathrm{P}=40$, then $\mathrm{N}=365$ and if $\mathrm{P}=45$, then $\mathrm{N}=380$, consequently the sample size was 400 .

\section{Statistical analysis}

The collected data were tabulated and analyzed using SPSS version 16 software (SPSS Inc., Chicago, Illinois, USA). Categorial data were presented as number and percentages, while quantitative data were expressed as mean \pm SD and range. $x^{2}$ test was used to analyze categorial variables and Student'st test was used to assess the difference among two independent groups regarding normally distributed variables. Correlations were assessed by Spearman's correlation coefficient (rho). The accepted level of significance in this work was $P$ value less than 0.05 .

\section{RESULTS}

\section{Demographic data}

The mean age of women in group I (CS) and group II (VD) was $36.22 \pm 5.4$ and $37.14 \pm 6.62$ years, respectively, with insignificant difference between both the studied groups $(P=0.06)$. The majority of participating women had been married for less than 5 years $(72 \%$ in group I vs. $55.5 \%$ in group II). The mean duration of marriage was $5.73 \pm 2.43$ years in group I versus $6.97 \pm 2.66$ years in group II with statistically significant difference between both groups $(P<0.001)$. In relation to postpartum changes in body satisfaction, $51.5 \%$ of women in group I were unsatisfied compared with $33 \%$ in group II with significant difference between both groups $(P<0.001)$. Mediolateral episiotomy was performed to $95.5 \%(181 / 200)$ of women in group II.

\section{Women postpartum sexual activity}

Women in group I showed a significant higher score $(P<0.05)$ in all items of FSFI except in orgasm compared with group II (Table 1). Furthermore, women who had episiotomy in group II experienced insignificant $(P>0.05)$ reduction in the mean score of all subdomains(desire, arousal, lubrication, orgasm, and total FSFI) except in satisfaction and pain domains as they showed a significant reduction ( $P=0.035$ and 0.04 , respectively) in relation to their counterparts (VD without episiotomy) in the same group (Table 2). From the 400 studied women, 206 (51.5\%) had FSD ( $\leq 26.55)$, where $125(60.7 \%)$ were in group II and $81(39.3 \%)$ in group I with a significant difference between both studied groups $(P<0.001)$. From group I more than half $(63 \%)$ of the women having SD were multiparous $(51 / 81)$, likewise in group II multiparous represented $54.4 \%(68 / 125)$. Age was inversely correlated $(P>0.05)$ to all subdomains except for the desire domain $(\mathrm{r}=0.023, P=0.64)$. Duration of marriage also disclosed a negative insignificant $(P>0.05)$ correlation to all subdomains except for arousal domain $(\mathrm{r}=-0.16$, $P=0.021)$. Body change satisfaction was negatively and positively correlated $(P>0.05)$ to two (desire and arousal) and five subdomains (lubrication, orgasm, satisfaction, pain, and total FSFI), respectively. The level of education also showed insignificant negative and positive correlation to two (arousal and pain) and five subdomains (desire, lubrication, orgasm, satisfaction, and total FSFI), respectively.

\section{Husbands' postpartum sexual activity}

Husbands of women in group I (CS) had a significant $(P<0.05)$ higher mean score in three domains (sexual desire, intercourse, and overall satisfaction) as well as in total IIEF score when compared with those in group II. On 
the other hand, the mean score of orgasmic function was significantly $(P<0.05)$ higher in men of group I compared with those in group II (Table 1). Moreover, all subdomains of IIEF were insignificantly $(P>0.05)$ declined in spouses of women who had mediolateral episiotomy during their last childbirth in relation to their equivalents of the same group (Table 2).

The following was observed on correlating different items of FSFI to IIEF: female arousal domain was significantly correlated to three male domains (erectile function, intercourse satisfaction, and total score of IIEF); female orgasmic domain was correlated to male overall satisfaction; and the total FSFI was correlated to male overall and intercourse satisfaction plus total IIEF (Table 3).

Neither duration of marriage, the level of education, nor females' body change satisfaction had a significant correlation to IIEF.

Table 1: Comparison of the mean values of female sexual function indexand international index of erectile function domains in women and their husband's postpartum in both studied groups.

\begin{tabular}{|c|c|c|c|c|c|c|}
\hline \multirow[b]{2}{*}{ FSFI domains } & \multicolumn{2}{|c|}{$\mathrm{CS}$} & \multicolumn{2}{|c|}{ VD } & \multirow[b]{2}{*}{ Student $\mathrm{t}$ test } & \multirow[b]{2}{*}{$P$ value } \\
\hline & Mean \pm SD & Range & Mean \pm SD & Range & & \\
\hline Desire & $4.0 \pm 1.10$ & $1.2-6$ & $3.53 \pm 0.93$ & $1.2-6$ & 4.65 & $<0.001^{*}$ \\
\hline Arousal & $4.39 \pm 0.92$ & $1.2-6$ & $4.10 \pm 1.14$ & $1.2-6$ & 2.76 & $0.006^{*}$ \\
\hline Lubrication & $4.65 \pm 1.0$ & $1.2-6$ & $4.25 \pm 1.14$ & $1.2-6$ & 3.75 & $<0.001^{*}$ \\
\hline Orgasm & $4.43 \pm 0.87$ & $1.2-6$ & $4.34 \pm 1.05$ & $1.2-6$ & 0.89 & 0.37 \\
\hline Satisfaction & $5.06 \pm 0.98$ & $1.2-6$ & $4.85 \pm 0.95$ & $1.2-6$ & 2.22 & $0.027^{*}$ \\
\hline Pain & $4.14 \pm 1.27$ & $1.2-6$ & $3.79 \pm 1.29$ & $1.2-6$ & 2.75 & $0.006^{*}$ \\
\hline \multirow[t]{2}{*}{ Total FSFI } & $26.67 \pm 4.14$ & $7.2-36$ & $24.85 \pm 4.30$ & $14.2-34.2$ & 4.30 & $<0.001^{*}$ \\
\hline & \multicolumn{2}{|c|}{ Husbands of women in group I (CS) } & \multicolumn{2}{|c|}{ Husbands of women in group II (VD) } & & \\
\hline IIEF domains & Mean \pm SD & Range & Mean \pm SD & Range & Student $t$ test & $P$ value \\
\hline Erectile function & $26.14 \pm 4.36$ & $13-30$ & $25.82 \pm 4.40$ & $12-30$ & 0.73 & 0.47 \\
\hline Orgasmic function & $8.45 \pm 1.69$ & $4-10$ & $8.81 \pm 1.35$ & $5-10$ & 2.36 & $0.019^{*}$ \\
\hline Sexual desire & $7.77 \pm 1.22$ & $4-10$ & $7.49 \pm 1.33$ & $4-10$ & 2.16 & $0.032^{*}$ \\
\hline Intercourse satisfaction & $12.18 \pm 1.68$ & $7-15$ & $11.07 \pm 1.93$ & $7-15$ & 6.17 & $<0.001^{*}$ \\
\hline Overall satisfaction & $8.94 \pm 1.13$ & $6-10$ & $8.30 \pm 1.42$ & $4-10$ & 4.98 & $<0.001^{*}$ \\
\hline Total IIEF & $63.47 \pm 7.91$ & $40-74$ & $61.48 \pm 7.07$ & $44-74$ & 2.65 & $0.008^{*}$ \\
\hline
\end{tabular}

CS, cesarean section; FSFI, female sexual function index; IIEF, international index of erectile dysfunction; VD, vaginal delivery.

*Statistically significant difference $P$ value less than 0.05 .

Table 2: Effect of episiotomy on couples' sexual function among women with normal vaginal delivery

\section{FSFI}

\begin{tabular}{|c|c|c|c|c|}
\hline Episiotomy & $\begin{array}{c}\text { Vaginal delivery } \\
\text { with mediolateral } \\
\text { episiotomy }(181 / 200)\end{array}$ & $\begin{array}{c}\text { Vaginal delivery } \\
\text { with mediolateral } \\
\text { episiotomy }(181 / 200)\end{array}$ & Student'st test & $P$ value \\
\hline Desire & $3.52 \pm 0.92$ & $3.54 \pm 1.0$ & 0.06 & 0.95 \\
\hline Arousal & $4.10 \pm 1.13$ & $4.14 \pm 1.28$ & 0.14 & 0.89 \\
\hline Lubrication & $4.23 \pm 1.14$ & $4.45 \pm 1.11$ & 0.81 & 0.42 \\
\hline Orgasm & $4.35 \pm 1.06$ & $4.23 \pm 1.03$ & 0.47 & 0.64 \\
\hline
\end{tabular}




\begin{tabular}{|c|c|c|c|c|}
\hline Satisfaction & $4.89 \pm 0.94$ & $4.42 \pm 0.90$ & 2.07 & $0.04 *$ \\
\hline Pain & $3.72 \pm 1.28$ & $4.38 \pm 1.32$ & 2.12 & $0.035^{*}$ \\
\hline Total FSFI & $24.82 \pm 4.34$ & $25.16 \pm 3.97$ & 0.33 & 0.75 \\
\hline \multicolumn{5}{|c|}{ FSFI } \\
\hline Episiotomy & $\begin{array}{c}\text { Vaginal delivery } \\
\text { with mediolateral } \\
\text { episiotomy }(181 / 200)\end{array}$ & $\begin{array}{c}\text { Husbands' of women } \\
\text { without episiotomy } \\
(19 / 200)\end{array}$ & Student'st test & $P$ value \\
\hline Desire & $($ mean \pm SD $)$ & $($ mean \pm SD $)$ & & \\
\hline Arousal & $25.72 \pm 4.43$ & $26.74 \pm 4.04$ & 0.96 & 0.34 \\
\hline Lubrication & $8.79 \pm 1.33$ & $8.95 \pm 1.51$ & 0.48 & 0.63 \\
\hline Orgasm & $7.47 \pm 1.33$ & $7.68 \pm 1.34$ & 0.67 & 0.51 \\
\hline Satisfaction & $11.03 \pm 1.93$ & $11.37 \pm 1.95$ & 0.72 & 0.47 \\
\hline Pain & $8.28 \pm 1.45$ & $8.47 \pm 1.22$ & 0.57 & 0.57 \\
\hline Total FSFI & $61.29 \pm 7.08$ & $63.21 \pm 6.91$ & 1.13 & 0.26 \\
\hline
\end{tabular}

FSFI, female sexual function index; IIEF, international index of erectile dysfunction.

*Statistically significant difference $P$ value less than 0.05 .

Table 3: Correlation between male and female sexual functions among the studied groups.

\begin{tabular}{|c|c|c|c|c|c|c|c|c|c|c|c|c|}
\hline \multirow[b]{3}{*}{ IIEF } & \multicolumn{10}{|c|}{ FSFI } & & \\
\hline & \multicolumn{2}{|c|}{ Erectile function } & \multicolumn{2}{|c|}{ Orgasmic function } & \multicolumn{2}{|c|}{ Sexual desire } & \multicolumn{2}{|c|}{$\begin{array}{l}\text { Intercourse } \\
\text { satisfaction }\end{array}$} & \multicolumn{2}{|c|}{$\begin{array}{c}\text { Overall } \\
\text { satisfaction }\end{array}$} & \multicolumn{2}{|c|}{ Total IIEF } \\
\hline & Rho & $P$ & Rho & $P$ & Rho & $P$ & Rho & $P$ & Rho & $P$ & Rho & $P$ \\
\hline Desire & -0.038 & 0.44 & -0.09 & 0.077 & 0.034 & 0.50 & 0.097 & 0.052 & 0.09 & 0.08 & 0.005 & 0.92 \\
\hline Arousal & 0.16 & $0.001^{* *}$ & 0.09 & 0.08 & 0.045 & 0.37 & 0.14 & $0.005^{* *}$ & 0.07 & 0.18 & 0.17 & $0.001^{* *}$ \\
\hline Lubrication & 0.06 & 0.20 & 0.05 & 0.30 & 0.08 & 0.14 & 0.07 & 0.16 & 0.72 & 0.15 & 0.09 & 0.071 \\
\hline Orgasm & 0.008 & 0.87 & 0.002 & 0.97 & 0.08 & 0.13 & 0.08 & 0.09 & 0.14 & $0.007^{* *}$ & 0.06 & 0.21 \\
\hline Satisfaction & 0.038 & 0.45 & 0.007 & 0.89 & 0.097 & 0.053 & 0.047 & 0.34 & 0.09 & 0.08 & 0.07 & 0.18 \\
\hline Pain & 0.013 & 0.80 & -0.045 & 0.37 & -0.033 & 0.51 & 0.09 & 0.08 & 0.07 & 0.17 & 0.04 & 0.40 \\
\hline Total FSFI & 0.06 & 0.23 & 0.002 & 0.97 & 0.07 & 0.18 & 0.15 & $0.003^{* *}$ & 0.13 & $0.012^{*}$ & 0.11 & $0.035^{*}$ \\
\hline
\end{tabular}

FSFI, female sexual function index; IIEF, international index of erectile function; rho, Spearman's correlation coefficient.

**Significant difference $P$ value less than 0.05 . 


\section{DISCUSSION}

FSD affects $41 \%$ of women worldwide,especially in the age range between 18 and 44 years. Factors affecting femalesexual functionare variable and vary from one country to another ${ }^{[6]}$.

Although, pregnancy and delivery have different effects on body organs, especially on the genitalia and pelvic floor muscles, which can cause $\mathrm{SD}^{[7]}$; up till now, there is controversy over the impact of the mode of delivery on female sexual function and there are limited data concerning the sexual relationship of each partner after childbirth in a heterosexual couple. Therefore, this study was conducted to assess and compare the impact of mode of delivery on sexual function of married couples and explore the relationship between female and male sexual functions after birth

This study enrolled 400 couples. They were equally divided according to the mode of delivery into two groups: group I (200) included women who had CS and group II (200) included those whohad VD and their husbands.

Although, the current study in addition to Saleh et al. ${ }^{[8]}$ studyshowed that the CS group had significantly higher score in total FSFI and almost all subdomains when compared withthe VD group, Kahramanoglu et al..$^{[9]}$ stated that irrespective to the mode of delivery, sexual functions within 6 months after delivery will be comparable to that of before pregnancy. This discrepancy is most probably due to methodological differences, as they used a face-to-face questionnaire 3-6 months postpartum and at $12-24$ months. The questionnaire was discussed over the telephone, unlike the current study wherethe identity of the participants was kept anonymous and the information they supplied were secured.

Although all sexual domains declined in those who underwent episiotomy, arousal and lubrication were the only domains which showed a significant decline in relation to their equivalents in the same group. This was in harmony with the Barbara et al. ${ }^{[10]}$ study, who concluded that episiotomy is usually coupled with poor sexual function; in addition, another study ${ }^{[1]}$ stated that its effect can persist up to 5 years postpartum. Likewise, De Souza et al. ${ }^{[12]}$ specified arousal as the most affected domain. On the other hand, Barbara et al. ${ }^{[10]}$ specified orgasm as the most affected domain. This difference was due to different inclusion criteria where they enrolled only primiparous women in contrast to the current study which enrolled both.

Almost $52 \%$ of participating women inthis study had FSD, which can be attributed to sleep disturbance, physical discomfort, and uncooperative husband ${ }^{[13]}$. SD was specifically detected more in women of group II relative to those of group I, which was also detected in the Saydam et al. ${ }^{[14]}$ study, where the latter showed a higher percentage of SD among postpartum women since they enrolled nursing women. Moreover, de Araujo et al. ${ }^{[15]}$ affirmed that VD causes more levator ani muscle injuries than CS as determined by three-dimensional ultrasound evaluation. Knowing that contraction of levator ani muscle is important in enhancing sexual response as it causes elevation and straightening of the uterus plus elongation and narrowing of the vagina ${ }^{[15]}$.

Furthermore, SD was also noticed in multiparous women more than nulliparous in both studied groups in this study. This is in harmony with the Banaei et al. ${ }^{[2]}$ study; they showed that SD was lower in multiparous women compared with primiparous women. Also, Makkii and Yazdi $^{[7]}$ in their study clarified that vaginal looseness is one of the main reasons of SD among multiparous women and especially in women with VD.

Age and duration of marriage showed insignificant correlation to FSFI domains in contrast to Dennerstein et $a l^{[16]}$ and Banaei et $a l^{[17]}$ studies, respectively. This difference is because almost three quarters of the participating women were in their second decade and had been married for less than 5 years in contrast to previous studies.

To the best of our knowledge, this is the first study to evaluate men's sexual function according to the mode of delivery of their wives and to assess if women's SD has an influence on their spouses' sexuality.

In the current study, husbands of women in the CS group had a significant higher mean score in the total IIEF score when compared with their equivalents of the same group. This was in agreement with the Gungor et al. ${ }^{[18]}$ study, which showed that the mean of the overall male sexual function group according to the Golombock-Rust Inventory of Sexual Satisfaction was more in the CS group compared with the VD. Gray et al. ${ }^{[19]}$ and Abdel Rahman et al. ${ }^{[20]}$ also suggested that a fraction of men reported alterations in theirsexual behavior during a partner's postpartum period in the former study, while the latter study showed that partners' of women with urinary incontinence had asignificant decline in almost all IIEF when compared with the control group (partners of women with no urinary incontinence). Both studies showed that such decline was commonly aligned with those of a partner. This alignment was also detected in our study on correlating different items of FSFI to IIEF.

In this study, female's arousal domain was significantly correlated to male intercourse satisfaction and total IIEF, which was also detected in the Hendrickx et al. ${ }^{[21]}$ and Khalesi et al. ${ }^{[22]}$ studies, respectively. Sexual impairment was assessed using the sexual functioning scale in the former study, while the latter used FSFI and IIEF on pregnant women. Moreover, the total FSFI was correlated to overall and intercourse satisfaction plus total IIEF in this study along with the Khalesi et al. ${ }^{[22]}$ study, which assessed the effects of pregnancy on sexual function of couples during their prenatal visits. Erectile function showed 
no relation to the mean score of subdomains of FSFI neither in this study nor in another study ${ }^{[23]}$, which used a community-based survey. In addition, our study was also in partial agreement with another study ${ }^{[24]}$ as they concluded that there was a significant correlation to all IIEF domains with the exception of male erectile function with all FSFI. The previous study evaluated SD in female partners of men who have undergone radical prostatectomy.

\section{LIMITATIONS OF THE STUDY}

Our study had some limitations, as we didnot ask about the type of previous deliveries in multiparous women and the baby's birth weight. We also believe that using a validated questionnaire as well as assessing sexual relationship between couples gives more strength to our work.

\section{RECOMMENDATION}

It is recommended to limit routine use of episiotomy unless recommended, especially with multiparous women. Appropriate sexual education of both couples is required after childbirth regardless of the mode of delivery to avoid or minimizeSD and to improve marital satisfaction and intimacy.

\section{ACKNOWLEDGEMENTS}

The authors acknowledge their patients and their partners as this work would have never been possible without their cooperation.

With the submission of this manuscript, we confirm that: all authors of this research paper have directly participated in the planning, execution, or analysis of this study. All authors of this paper have read and approved the final version submitted.

\section{CONFLICT OF INTEREST}

There are no conflicts of interest

\section{REFERENCES}

1. Garthus-NiegelS, HorschA, HandtkeE, von SoestT, AyersS, WeidnerK, Eberhard-GranM. The impact of postpartum posttraumatic stress and depression symptoms on couples' relationship satisfaction: a population-based prospective study. Front Psychol2018; 9:1728.

2. BanaeiM, AlidostF, GhasemiE, DashtiS. A comparison of sexual function in primiparous and multiparous women. J Obstet Gynaecol2019; 40:1-8.

3. AnisTH, GheitSA, SaiedHS, Al kherbashSA.
Arabic translation of female sexual function index and validation in an Egyptian population. J Sex Med2011;8:3370-3378.

4. RosenR, BrownC, HeimanJ, LeiblumS, MestonCM, ShabsighR, et al. The female sexual function index (FSFI): a multidimensional self-report instrument for the assessment of female sexual function. J Sex Marital Ther2000; 26:191-208.

5. RosenRC, RileyA, WagnerG,OsterlohIH. The international index of erectile function (IIEF): a multidimensional scale for assessment of erectile dysfunction. Urology1997; 49:822-830.

6. McCool-MyersM, TheurichM, ZuelkeA, KnuettelH, ApfelbacherC. Predictors of female sexual dysfunction: a systematic review and qualitative analysis through gender inequality paradigms. BMC Womens Health2018; 18:108.

7. MakkiiM, YazdiNA. Sexual dysfunction during primiparous and multiparous women following vaginal delivery. Tanzan $\mathrm{J}$ Health Res2012; 14:263-268.

8. SalehDM, HosamF, MohamedTM. Effect of mode of delivery on female sexual function: a cross-sectional study. J Obstet Gynaecol Res2019; 45:1143-1147.

9. KahramanogluI, BaktirogluM, HamzaogluK, KahramanogluO, VeritFF, YucelO. The impact of mode of delivery on the sexual function of primiparous women: a prospective study. Arch Gynecol Obstet2017; 295:907-916.

10. BarbaraG, PifarottiP, FacchinF, CortinovisI, DridiD, RonchettiC, et al. Impact of mode of delivery on female postpartum sexual functioning: spontaneous vaginal delivery and operative vaginal delivery vs. cesarean section. J Sex Med2016; 13:393-401.

11. DoğanB, Günİ, ÖzdamarÖ, YılmazA, MuhçuM. Long-term impacts of vaginal birth with mediolateral episiotomy on sexual and pelvic dysfunction and perineal pain. J Matern Fetal Neonatal Med2017; 30:457-460.

12. De SouzaA, DwyerPL, CharityM, ThomasE, FerreiraCH, SchierlitzL. The effects of mode delivery on postpartum sexual function: a prospective study. BJOG2015; 122:1410-1418.

13. KoSH, ChenCH, WangHH, SuYT. Postpartum women's sleep quality and its predictors in Taiwan. J Nurs Scholarsh2014; 46:74-81.

14. SaydamBK, Demireloz AkyuzM, SogukpinarN, 
Ceber TurfanE. Effect of delivery method on sexual dysfunction. J Matern Fetal Neonatal Med2019; $32: 568-572$.

15. de AraujoCC, CoelhoSA, StahlschmidtP, JuliatoCRT. Does vaginal delivery cause more damage to the pelvic floor than cesarean section as determined by 3D ultrasound evaluation? A systematic review. Int Urogynecol J2018; 29:639-645.

16. DennersteinL, GuthrieJR, HayesRD, DeRogatisLR, LehertP. Sexual function, dysfunction, and sexual distress in a prospective, population-based sample of mid-aged, Australian-born women. J Sex Med2008; 5:2291-2299.

17. BanaeiM, MoridiA, DashtiS. Sexual dysfunction and its associated factors after delivery: longitudinal study in iranian women. Mater Sociomed2018; 30:198-203.

18. GungorS, BaserI, CeyhanT, KarasahinE, KilicS. Does mode of delivery affect sexual functioning of the man partner?J Sex Med2008; 5:155-163.

19. GrayPB, ReeceJA, Coore-DesaiC, DinnallJohnsonT, PellingtonS, Samms-VaughanM. Sexuality among fathers of newborns in Jamaica.
BMC Pregnancy Childbirth2015; 15:44.

20. Abdel RahmanSH, Abou TalebA, Abou ZeidAM,El DesoukyRS.Do female urinary incontinence subtypes have different effects on the sexual life of couples?Sex Relationship Ther2019; 34:1-11.

21. HendrickxL, GijsL, JanssenE, EnzlinP. Predictors of sexual distress in women with desire and arousal difficulties: distinguishing between personal, partner, and interpersonal distress.J Sex Med2016; 13:1662-1675.

22. KhalesiZB, BokaieM, AttariSM. Effect of pregnancy on sexual function of couples.Afr Health Sci2018; 18:227-234.

23. ZhangH, FanS, YipP. The association between female sexual dysfunction and the husband's erectile dysfunction: evidence from married couples in Hong Kong. J Sex Marital Ther2016; 42:214-222.

24. 24. ShindelA, QuayleS, YanY, HusainA, NaughtonC. Sexual dysfunction in female partners of men who have undergone radical prostatectomy correlates with sexual dysfunction of the male partner. J Sex Med2005; 2:833-841. 